\title{
Interventions Improve Gait Regularity in Patients with Peripheral Neuropathy While Walking on an Irregular Surface Under Low Light
}

\author{
James K. Richardson, MD, ${ }^{*}$ Sibylle B. Thies, $M S,{ }^{\dagger}$ Trina K. DeMott, MS, PT, ${ }^{*}$ and \\ James A. Ashton-Miller, $\mathrm{PhD}^{+}$
}

OBJECTIVES: To determine which, if any, of three inexpensive interventions improve gait regularity in patients with peripheral neuropathy (PN) while walking on an irregular surface under low-light conditions.

DESIGN: Observational.

SETTING: University of Michigan Biomechanics Research Laboratory.

PARTICIPANTS: Forty-two patients with PN (20 women), mean age \pm standard deviation $=64.5 \pm 9.7$.

INTERVENTIONS: A straight cane, touch of a vertical surface, or semirigid ankle orthoses.

MEASUREMENTS: Step-width variability and range, step-time variability, and speed.

RESULTS: Subjects demonstrated significantly less stepwidth variability $($ mean $=41.0 \pm 1.5,36.9 \pm 1.6,37.2 \pm$ 1.3 , and $35.9 \pm 1.5 \mathrm{~mm}$ for baseline, cane, orthoses, and vertical surface, respectively; $P<.0001)$ and range $(182.7 \pm$ $7.4,163.7 \pm 8.3,164.3 \pm 7.4,154.3 \pm 6.9 \mathrm{~mm}$ for baseline, cane, orthoses and vertical surface, respectively; $P=$ .0006) with each of the interventions than under baseline conditions. Step-time variability significantly decreased with use of the orthoses and vertical surface but not the cane $(P=.0001)$. Use of a cane, but not orthoses or vertical surface, was associated with decreased speed $(0.79 \pm 0.03$, $0.73 \pm 0.03,0.79 \pm 0.03,0.80 \pm 0.03 \mathrm{~m} / \mathrm{s}$ for baseline, cane, orthoses, and vertical surface, respectively; $P=.0001$ ). CONCLUSION: Older patients with PN demonstrate improved spatial and temporal measures of gait regularity with the use of a cane, ankle orthoses, or touch of a vertical surface while walking under challenging conditions. The decreased speed and stigma associated with the cane and uncertain availability of a vertical surface suggest that the

From the Departments of *Physical Medicine and Rehabilitation and ${ }^{\dagger}$ Mechanical Engineering, University of Michigan, Ann Arbor, Michigan.

Funding was received from Public Health Service Grants K23 AG 00989 (JKR) and P60 AG 08808 (SBT, TKD, JAAM).

Address correspondence to James K. Richardson, MD, Associate Professor, University of Michigan, Department of Physical Medical and Rehabilitation, Medical Professional Building D5200, Ann Arbor, MI 48109. E-mail: jkrich@umich.edu ankle orthoses may be the most practical intervention. J Am Geriatr Soc 52:510-515, 2004.

Key words: neuropathy; gait; balance; orthoses; assistive device

$T$ he human method of bipedal ambulation is deceptively demanding because of the need to propel a high center of body mass over a narrow and variable base of support. The central nervous system requires timely and accurate information to make the motor adjustments necessary to maintain balance. Distortion of this afferent flow of information often leads to impairments in balance and falls. Peripheral neuropathy (PN) diminishes somatosensory input from the distal lower extremities and, accordingly, has been found to adversely affect lower extremity function in older women ${ }^{1}$ and to be associated with falls. Increased fall rates have been found in subjects with purely diabetic PN, ${ }^{2}$ as well as in subjects with PN due to a variety of etiologies. ${ }^{3}$ Even leprosy, the most common cause of PN in the past, was likely a cause of falls, given that the fracture rate in a medieval leprosy hospital skeletal sample was four times that in a control sample. ${ }^{4}$ The high prevalence of diabetes mellitus and impaired glucose tolerance in people aged 60 to $74,{ }^{5}$ in conjunction with a $7.1 \%$ prevalence of $\mathrm{PN}$ in normoglycemic persons, ${ }^{6}$ suggests that the prevalence of $\mathrm{PN}$ in this age group is approximately $22 \%$.

The majority of falls occur during ambulation, often in association with surface irregularities or decreased visual input. ${ }^{7}$ Lateral falls are common ${ }^{8}$ and particularly costly in socioeconomic terms because of the increased likelihood of hip fracture. ${ }^{9}$ Medial-lateral stability during ambulation is of clinical importance ${ }^{10}$ and is controlled most effectively ${ }^{11}$ and efficiently ${ }^{12}$ through step-width modification via higher neural centers. ${ }^{13}$ Increased variability of gait parameters have been found to be associated with falls ${ }^{14,15}$ and correlated with severity of central ${ }^{16-18}$ and peripheral neurological diseases (unpublished data). Taken together, these studies suggest that variability in gait parameters in 
general are associated with falls and disease severity and that control of step width is critical to frontal plane stability.

Therefore, in this study, patients with PN were analyzed while they walked on an irregularly surfaced walkway under low-light conditions. Outcomes included step-width and step-time variabilities (as determined by respective standard deviations (SDs) of these measures), step-width range (as determined by the difference between maximum and minimum step widths), and speed. Interventions were chosen to improve frontal plane balance and included a cane, ankle orthoses that provided medial/lateral support, and touch of a vertical surface. It was hypothesized that patients with PN using one or more of the interventions would demonstrate changes in gait parameters consistent with improved dynamic frontal-plane balance and step control (decreased step-width and step-time variabilities and decreased step-width range), compared with the baseline condition without these interventions.

\section{METHODS}

\section{Subjects}

Patients with PN were recruited from the University of Michigan Electrodiagnostic Laboratory and Physical Medicine and Rehabilitation Outpatient Orthotics and Prosthetics Clinic. All patients underwent history, physical examination, and electrodiagnostic testing. The University of Michigan institutional review board approved the project, and all subjects gave written informed consent.

Inclusion criteria were age between 45 and 85, symptoms consistent with PN, ability to speak and understand English, ability to ambulate household distances without an assistive device, physical examination consistent with PN (absent or relatively decreased Achilles reflexes, and decreased distal lower extremity sensation (vibration, pinprick, and light touch) that improved proximally), and electrodiagnostic evidence of a diffuse, primarily axonal polyneuropathy as evidenced by: sural responses (absent or decreased amplitude $(<6 \mu \mathrm{V})$ with a normal or minimally prolonged distal latency $(<5.0 \mathrm{~ms})$ stimulating $14 \mathrm{~cm}$ proximally from the recording site posterior to the lateral malleolus) and peroneal or tibial motor responses (absent or decreased in amplitude $(<2 \mathrm{mV}$ for peroneal and $<3 \mathrm{mV}$ for tibial) with a normal distal latency $(<6.2 \mathrm{~ms}$ stimulating $9 \mathrm{~cm}$ and $8 \mathrm{~cm}$ proximally from recording sites over the extensor digitorum brevis and abductor hallicus muscles, respectively).

If bilateral sural responses were absent, then motor responses were not always performed. If the sural responses were present but reduced in amplitude, then motor responses were required and must also have been of reduced amplitude. Needle examination was normal or demonstrated findings consistent with PN. The subjects were also evaluated using the Michigan Diabetes Neuropathy Score (MDNS). The MDNS, a 0- to 46-point scale (higher score reflecting more-severe PN) that correlates well with more-extensive neuropathy staging scales, ${ }^{19}$ was used as a clinical measure of PN severity, not as an inclusion/ exclusion criterion.

Exclusion criteria were weight greater than 300 pounds $(136 \mathrm{~kg})$; evidence on physical examination of central neurological dysfunction; musculoskeletal abnormality, such as severe scoliosis or amputation; and electrodiagnostic evidence of a lower extremity abnormality other than $\mathrm{PN}$, such as a myopathic process or radiculopathy.

\section{Subject Preparation and Experimental Apparatus}

Subjects wore flat-soled athletic shoes supplied by the laboratory. Subjects were placed in a safety harness secured to an overhead track. The harness suspension was adjusted so as to prevent the knees from coming into contact with the floor when the subject hung unsupported.

A 1.5- by 10-meter piece of industrial carpet was modified to create an irregular surface by randomly arranging prismshaped pieces of wood (height $=1.5 \mathrm{~cm}$, width $=3.5 \mathrm{~cm}$, length $=6-16 \mathrm{~cm}$ ) beneath the mid $6.5-\mathrm{m}$ section of the carpet at a density of 26 pieces $/ \mathrm{m}^{2}$ (Figure 1). Low-light conditions (average 50 lux) were maintained by dimming lights in the room. Two optoelectronic markers (infraredemitting diodes) were placed $5 \mathrm{~cm}$ apart on a malleable
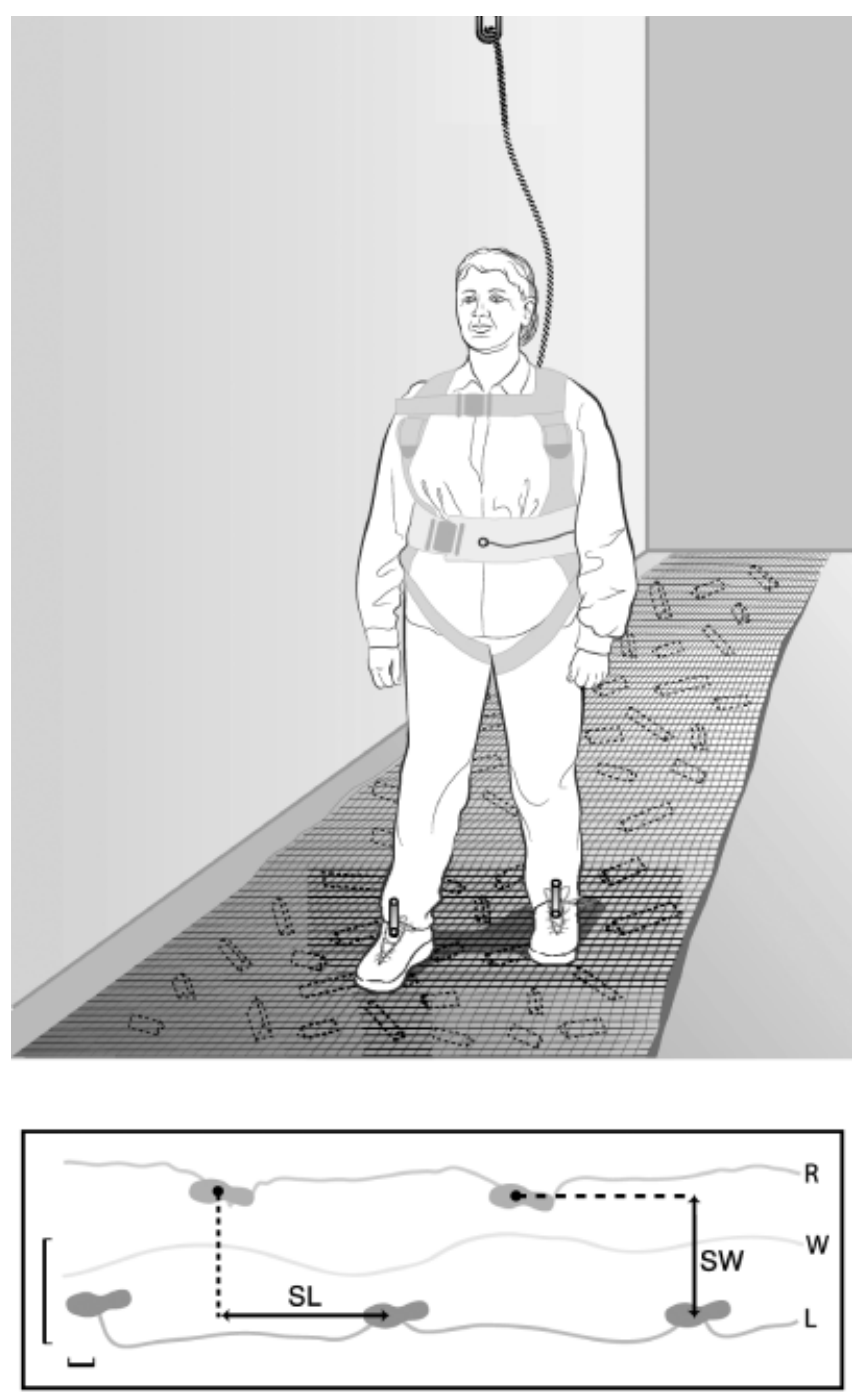

Figure 1. Schematic of irregular surface (randomly oriented triangular prisms are shown beneath the carpet surface) and placement of optoelectronic markers on the body for calculation of step width, step length, and velocity. 
aluminum strip $(10 \mathrm{~cm} \times 1.5 \mathrm{~cm})$ inserted under the tongue of each shoe. The top marker was located anterior to the center of the malleoli. A marker was also placed on a belt in the midline at the level of the umbilicus. Two foot switches, each a force-sensing resistor, were placed underneath the insole of each shoe. One switch was placed under the first metatarsophalangeal joint and the other beneath the calcaneus. Double support was defined as the period of time in the gait cycle during which at least one switch inside each shoe was activated. Kinematic data were measured at $100 \mathrm{~Hz}$ using an optoelectronic camera system (Optotrack 3020, Northern Digital Corp, Waterloo, Ontario, Canada) toward which the subject walked within the boundaries of the walkway.

\section{Interventions}

The interventions were administered under the supervision of an experienced physical therapist (TD). The cane height was adjusted so that the handle was at the wrist crease when the subject's arms hung in a relaxed fashion at the side. ${ }^{20}$ Subject were taught to use the cane with their nondominant upper extremity and to place the cane on the ground in synchrony with the contralateral lower extremity. The orthoses (Figure 2, Active Ankle Systems Inc., Louisville, KY) were placed on each ankle per manufacturer instructions, with the foam-lined shells oriented on the medial and lateral aspects of the ankle and lower leg and held in place with hook and loop straps. Subject were instructed to use their upper extremity to touch the vertical surface, which was made of dense insulating foam supported on metal struts, at approximately shoulder height during ambulation. Subjects used the palmar or dorsal surface of their hands, depending on their preference. A 5-minute period of practice with the each of the interventions, in a well-lighted hall with a linoleum floor, was allowed before testing.
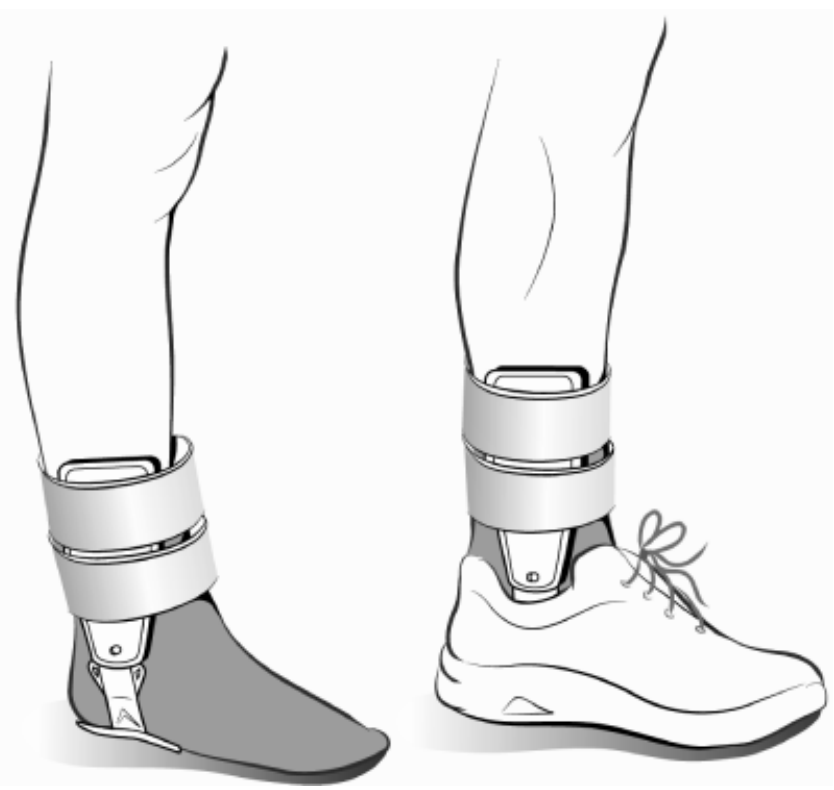

Figure 2. The ankle orthoses in place, with foam-lined shells on the medial and lateral aspects of the lower leg.

\section{Order of Testing}

The subjects first performed 10 trials (each trial walking down the irregular surface and back) without intervention. Subjects then performed 10 trials of each intervention in a prearranged randomized order, followed by 10 more trials without intervention to control for fatigue or practice effects. Before each set of trials, subjects were instructed to walk at their own comfortable pace, "as if they were walking to mail a letter.”

\section{Gait/Data Analysis}

The kinematic data were processed using a custom algorithm written in MATLAB to quantify step width, step length, and walking speed. Speed was calculated by taking the time derivative of the waist marker during what was defined as the "comfortable gait speed" interval. This interval was found by excluding data taken when the waist marker velocity was less than $85 \%$ of the maximum velocity for that trial, to eliminate steps taken while the subject accelerated to and decelerated from the comfortable gait speed interval. Similarly, the other gait parameters were only included in the analysis during this interval. Step time was calculated from the closure of one of the two switches in each shoe. Step width and step length were defined as the medial-lateral and anterior-posterior distance, respectively, between ankle markers during double support (Figure 1). The SD and range-of-gait parameters served as measures of variability. Step-width range was determined by subtracting the minimum step width from the maximum step width for each subject under each condition. The mean number of steps analyzed per subject was $49.4,40.5,42.3,44.3$, and 44.8 for the first baseline, cane, orthosis, vertical surface, and second baseline conditions, respectively.

\section{Statistical Analysis}

Statistical analyses were performed using SAS version 8.2 (SAS Institute, Inc., Cary, NC). Means and SDs of gait parameters for the intervention trials were entered without modification, but gait parameters for the baseline condition were entered as the mean of the two baseline trials to control for practice and fatigue effects. Age was entered as continuous variable, but body mass index ((BMI) weight in $\mathrm{kg} /$ height in $\mathrm{m}^{2}$ ) and $\mathrm{PN}$ severity were entered as dichotomous variables (using $31.0 \mathrm{~kg} / \mathrm{m}^{2}$ and an MDNS of 18 , respectively, as cutoffs) to avoid excessive influence from outliers that occurred when entered as continuous variables. Repeated measures analysis of variance was performed to determine whether differences in gait parameters were present with respect to the intervention trials. Sex, BMI group, PN group, and age were used as covariates. Intervention-by-sex, intervention-by-PN group, intervention-by-BMI group, and BMI group-by-sex interactions were also inserted into the model. When statistically significant differences were found, model estimates of the means were compared, taking into account the appropriate variances and covariances. The resulting $P$-values were adjusted for multiple comparisons. Gait parameters from the pre- and postintervention baseline trials were compared using paired $t$ tests. For all analyses, a $P<.05$ was considered a significant difference, whereas $P \geq .05$ and $<.10$ was considered a trend. 


\section{RESULTS}

\section{Subjects}

Forty-four subjects met inclusion criteria. Two subjects (both women) dropped out because of fatigue; their data are not included. Therefore, 42 subjects ( 20 women) completed the protocol. The mean age, BMI, and MDNS scores \pm SD were $65.9 \pm 10.4,32.1 \pm 6.9$, and $17.6 \pm 5.5$, respectively. Thirty-three of the subjects had absent sural responses. Twenty-five of the subjects had MDNS scores of 18 or lower, and 17 subjects had scores greater than 18 , indicating more severe PN. The presumed causes of PN included diabetes mellitus $(n=30)$, idiopathic $(n=5)$, connective tissue disease $(n=3)$, toxic effect of medication $(n=2)$, hypothyroidism $(\mathrm{n}=1)$, and familial $(\mathrm{n}=1)$.

\section{Effects of Interventions}

The subjects demonstrated lower step-width variability with all three of the interventions than under baseline conditions. The three interventions were not significantly different from one another (Figure 3). Step-width range was also lower with each of the three interventions than under baseline conditions (Table 1). This decrease reached significance for the orthoses and vertical surface, whereas the cane demonstrated a trend toward decreased step-width range. The subjects demonstrated significantly lower step time variability when using the orthoses and the vertical surface than under the baseline condition or when using the cane. The baseline condition and cane did not differ with regard to step-time variability. Gait speed was significantly slower when using the cane. No significant differences in gait speed were present between the baseline condition, the orthoses, and vertical surface.

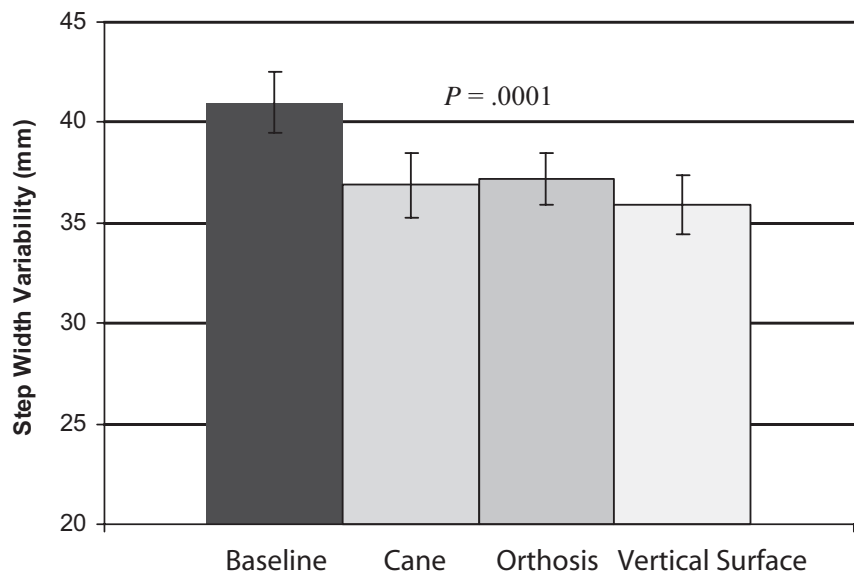

Figure 3. Model estimated means \pm standard errors were $41.0 \pm 1.5,36.9 \pm 1.6,37.2 \pm 1.3$, and $35.9 \pm 1.5 \mathrm{~mm}$ for control, cane, orthosis, and vertical surface, respectively $(P=.0001$, test of main effect). Post hoc comparisons demonstrated that all interventions significantly decreased step-width variability, compared with the baseline condition $(P=.0284$, .0024 , and .0001 for cane, orthosis, and vertical surface, respectively). No significant differences were identified when the interventions were compared with one another.

\section{Effects of Practice}

Practice effect was determined by comparing gait parameters from the first baseline trial to the second baseline trial. The subjects demonstrated significantly faster speed during the second baseline trial $(0.78 \pm 0.21$ vs $0.83 \pm 0.21$ $\mathrm{m} / \mathrm{s} ; P<.001)$ than during the first baseline trial but no change in step-width variability $(40.4 \pm 8.9$ vs $41.6 \pm$ $11.7 \mathrm{~mm} ; P=.244)$, step-time variability $(0.070 \pm 0.02$ vs $0.067 \pm 0.03$ seconds; $P=.319)$, or step-width range $(182.6 \pm 45.6$ vs $180.3 \pm 53.0 \mathrm{~mm} ; P=.702)$.

\section{Interactions Between Interventions and Age, BMI, PN Severity, and Sex}

Age, BMI, and PN severity did not interact with any of the interventions to influence any of the gait parameters, although sex interacted with the interventions to influence speed and step-time variability but not step-width variability or range. With regard to speed, men, but not women, walked significantly more quickly while using the orthosis than while using the cane $(P=.038$ for sex-by-intervention interaction and $P=.002$ for men using cane vs orthoses). The women walked faster using the orthoses than with the cane, but the difference was not significant. The women, but not the men, demonstrated less step-time variability when using the orthoses than when using the cane $(P=$ .0108 for sex-by-intervention interaction and $P=.054$ for women using cane vs orthoses).

\section{DISCUSSION}

\section{Summary of Major Findings}

The present study demonstrated that patients with PN were able to decrease their step-width variability, suggesting improved medial-lateral stability/control with the use of a cane, an ankle orthosis, or touch of a vertical surface. The interventions also decreased step-width range, a measure that represents the most extreme deviation in step width. A decrease in step-width range would be expected to decrease the likelihood of collisions between the swing and stance limbs, a common and destabilizing event in older persons in response to a lateral perturbation. ${ }^{21}$ In addition to decreasing step-width variability and range, the ankle orthosis and wall touch interventions led to significant decreases in step-time variability, a gait parameter that has been prospectively associated with falls. ${ }^{15}$ With the exception of the cane, which slowed the patients, these changes occurred without sacrificing speed.

\section{Theoretic Considerations and Potential Mechanisms of Action}

The data suggest that the interventions allowed for moreregular foot placement, both in the frontal plane and temporally. It is difficult to determine whether the wall touch and cane interventions provided improved afferent information, structural support, or both. Other work strongly suggests that upper extremity touch provides sensory information that influences balance ${ }^{22}$ and decreases medial-lateral trunk velocity of patients with PN during quiet standing. ${ }^{23}$ Alternatively, PN subjects who placed up to $25 \%$ of their body weight on a cane to maintain balance when performing unipedal stance during a perturbation 
Table 1. The Effect of Interventions on Gait Parameters

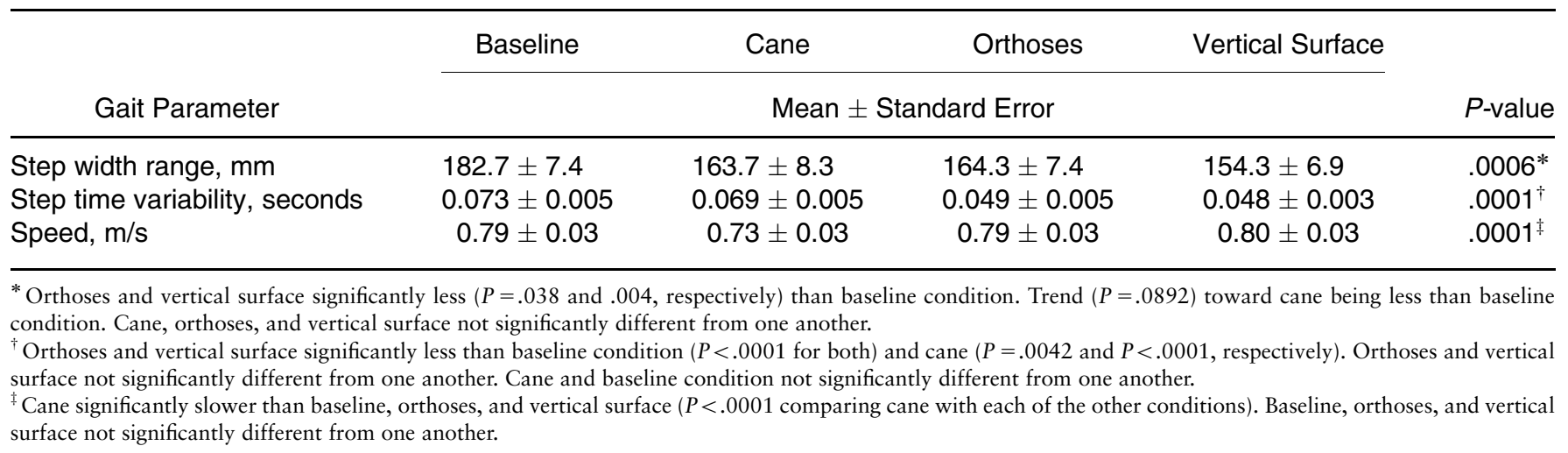

demonstrated the role of the cane in providing physical support. ${ }^{24}$ It is likely that the cane and wall interventions served dual functions - an afferent function that improved the patient's sense of trunk location in the frontal plane and an efferent function that provided structural support during occasions of significant loss of balance.

Similarly, the ankle orthoses may have provided improved support, sensory function, or both. The orthoses are designed to prevent ankle injuries in athletes by limiting extremes of ankle inversion and eversion and therefore likely stiffened the ankle in the frontal plane. This stiffening may have compensated for the known impairment in ankleinversion rate of torque development in patients with PN. ${ }^{25}$ This appears functionally relevant because the subtalar joint plays an active role in correcting errors in lateral sway $^{26}$ and medial-lateral foot placement. ${ }^{11}$ Furthermore, increased ankle stiffness has been found to decrease sway in response to a perturbation, ${ }^{27}$ and alteration of ankle torque was the initial adjustment to correcting postural errors during gait. ${ }^{28}$ Taken together, these findings suggest that improved subtalar stability has a dampening effect on medial-lateral perturbations. In further support of this, older women have demonstrated decreased sway and increased reach with a shoe with a $15-\mathrm{cm}$ collar. ${ }^{29}$ The orthoses may also have improved the known impairments in ankle inversion/eversion proprioceptive thresholds in patients with $\mathrm{PN}^{30}$ during swing or stance phase of gait, which in turn would allow adjustments to be made sooner. In support of this, other work suggests that speed of ankle response is critical to recovery from a perturbation and that older subjects demonstrate an improved postural response to perturbation with the use of an elevated edge, which provided negligible structural support, under the perimeter of the plantar foot. ${ }^{31}$

\section{Clinical Inferences}

These findings can influence the way healthcare practitioners educate patients with PN. It is rare for patients with $\mathrm{PN}$ with adequate vision to fall in locations that are well lit and have a floor surface that is firm, flat, and familiar to the patient. One study confirmed that patients with $\mathrm{PN}$ demonstrate stable dynamic balance during gait, by decreasing their speed, in the unchallenged situation. ${ }^{32}$ In such environments, all but the most severely impaired patients can likely manage without an assistive device, but when circumstances vary from these because of poor lighting, lack of familiarity, or a soft or irregular surface, then assistance is recommended.

The data suggest that the three interventions similarly improve balance in the frontal plane and so can provide this assistance, but there are some practical constraints. The obvious constraint for the vertical surface is uncertain availability. Although the cane is readily available, many patients are hesitant to use one for esthetic reasons. This may be circumvented by counseling the patient that the cane in this situation is an accommodation for an impaired special sense, analogous to the use of spectacles by those with impaired vision. The cane slowed the subjects significantly, which could be functionally relevant in situations where reasonable speed is necessary, such as crossing a broad avenue with a traffic light, ${ }^{33}$ but the subjects were given just 5 minutes of practice with each of the interventions, and it is possible that they might have increased their speed given longer practice with the cane. Finally, the cane requires the use of a hand, which limits the use of the upper extremities for carrying objects or for other purposes.

The ankle orthoses may be used anywhere, leaving the hands free, and did not cause the subjects to slow down. Nevertheless, a concern in patients with PN is the possibility of skin breakdown. Given the firm fit of the orthoses about the malleoli, skin breakdown could be of particular concern in patients with dependent edema or chronic venous stasis disease. This difficulty might be circumvented with the use of compressive stockings. Based on these findings, it is difficult to recommend having a patient with $\mathrm{PN}$ practice walking in a challenging situation. Although the data suggest that patients may improve their speed, there is no suggestion of improved stability with practice.

\section{Limitations and Conclusions}

The main limitation to this study is a lack of blinding and formal visual testing of the subjects. Blinding was not possible, given the nature of the interventions, but it seems likely that little bias was introduced given the fact that the outcomes were electronically quantified. Although the patients all had normal corrected vision by history, formal testing might have identified abnormalities. Because the patients were used as their own controls, any abnormalities identified would not change the major findings of the study, 
but there may have been vision-by-intervention interactions of clinical relevance that were not detected. In addition, the interventions were not tested in a real world setting, so the efficacy of the interventions during the initiation and termination of gait, change of direction, or distraction is uncertain.

Each of the interventions (a cane, touch of a vertical surface, and ankle orthoses) improves the gait regularity of patients with PN while walking on an irregular surface under low-light conditions. The interventions appeared to be equally effective, but the use of the cane was associated with significant slowing of gait speed. The interventions may allow PN patients to maintain function while minimizing fall risk under challenging circumstances.

\section{REFERENCES}

1. Resnick HE, Vinik AI, Schwartz AV et al. Independent effects of peripheral nerve dysfunction on lower-extremity physical function in old age. Diabetes Care 2000;23:1642-1647.

2. Cavanagh PR, Derr JA, Ulbrecht JS et al. Problems with gait and posture in neuropathic patients with insulin-dependent diabetes mellitus. Diabetes Med 1992;9:469-474.

3. Richardson JK. Factors associated with falls among older patients with diffuse polyneuropathy. J Am Geriatr Soc 2002;50:1767-1773.

4. Judd MA, Roberts CA. Fracture patterns at the Medieval Leper Hospital in Chichester. Am J Phys Anthropol 1998;105:43-55.

5. Harris MI, Flegal KM, Cowie CC et al. Prevalence of diabetes, impaired fasting glucose and impaired glucose tolerance in U.S. adults: The Third National Health and Nutrition Examination Survey, 1988-1994. Diabetes Care 1998;21:518-524.

6. Franklin GM, Kahn LB, Baxter J et al. Sensory neuropathy in non-insulindependent diabetes mellitus: The San Luis Valley Diabetes Study. Am J Epidemiol 1990;131:633-643.

7. Berg WP, Alessio HM, Mills EM et al. Circumstances and consequences of falls in independent community-dwelling older adults. Age Ageing 1997;26:261268.

8. Maki BE, Holliday PJ, Topper AK. A prospective study of postural balance and risk of falling in an ambulatory and independent elderly population. J Gerontol 1994;49:M72-M84.

9. Greenspan SL, Meyers ER, Maitland LA et al. Fall severity and bone mineral density as risk factors for hip fracture in ambulatory elderly. JAMA 1994;272:128-133.

10. Maki BE, McIlroy WE. Postural control in the older adult. Clin Geriatr Med 1996;12:635-658.

11. MacKinnon CD, Winter DA. Control of whole body balance in the frontal plane during human walking. J Biomech 1993;26:633-644.

12. Kuo AD. Stabilization of lateral motion in passive dynamic walking. Int J Robot Res 1999;18:917-930.
13. Bauby CE, Kuo AD. Active control of lateral balance in human walking. J Biomech 2000;33:1433-1440.

14. Maki BE. Gait changes in older adults. Predictors of falls or indicators of fear. J Am Geriatr Soc 1997;45:313-320.

15. Hausdorff JM, Rios DA, Edelberg HK. Gait variability and fall risk in community-living older adults: A 1-year prospective study. Arch Phys Med Rehabil 2001;82:1050-1056.

16. Charlett A, Weller C, Purkiss AG et al. Breadth of base whilst walking: Effect of ageing and parkinsonism. Age Ageing 1998;27:49-54.

17. Blin O, Ferrandez AM, Serratrice G. Quantitative analysis of gait in Parkinson patients: Increased variability of stride length. J Neurol Sci 1990; 98:91-97.

18. Hausdorff JM, Cudkowicz ME, Firtion R et al. Gait variability and basal ganglia disorders: Stride-to-stride variations of gait cycle timing in Parkinson's disease and Huntington's disease. Mov Disord 1998;13:428-437.

19. Feldman EL, Stevens MJ, Thomas PK et al. A practical two-step quantitative clinical and electrophysiological assessment for the diagnosis and staging of diabetic neuropathy. Diabetes Care 1994;17:1281-1289.

20. Sainsbury R, Mulley GP. Walking sticks used by the elderly. BMJ 1982; 284:1751.

21. Maki BE, Edmonstone MA, McIlroy WE. Age-related differences in laterally directed compensatory stepping behavior. J Gerontol A Biol Sci Med Sci 2000; 55A:M270-M277.

22. Jeka JJ. Light touch as a balance aid. Phys Ther 1997;77:476-487.

23. Dickstein R, Shupert CL, Horak FB. Fingertip touch improves postural stability in patients with peripheral neuropathy. Gait Posture 2001;14:238247.

24. Ashton-Miller JA, Yeh MWL, Richardson JK et al. A cane reduces loss of balance in patients with peripheral neuropathy: Results from a challenging unipedal balance test. Arch Phys Med Rehabil 1996;77:446-452.

25. Gutierrez MS, Helber MB, Dealva D et al. Mild diabetic neuropathy affects ankle motor function. Clin Biomech 2001;16:522-528.

26. Day BL, Steiger MJ, Thompson PD et al. Effect of vision and stance width on human body motion when standing: Implications for afferent control of lateral sway. J Physiol 1993;469:479-499.

27. Fitzpatrick RC, Taylor JL, McCloskey DI. Ankle stiffness of standing humans in response to imperceptible perturbation: Reflex and task-dependent components. J Physiol 1992;454:533-547.

28. Nashner LM. Balance adjustments of humans perturbed while walking J Neurophys 1980;44:650-664.

29. Lord SR, Bashford GM, Howland A et al. Effects of shoe collar height and sole hardness on balance in older women. J Am Geriatr Soc 1999;47: 681-684.

30. van den Bosch C, Gilsing MG, Lee SG et al. Peripheral neuropathy effect on ankle inversion and eversion detection thresholds. Arch Phys Med Rehabil 1995;76:850-856.

31. Maki BE, Perry SD, Norrie RG et al. Effect of facilitation of sensation from plantar foot-surface boundaries on postural stabilization in young and older adults. J Gerontol A Biol Sci Med Sci 1999;54A:M281-M287.

32. Dingwell JB, Cusumano JP, Stenrad D et al. Slower speeds in patients with diabetic neuropathy lead to improved local dynamic stability of continuous overground walking. J Biomech 2000;33:1269-1277.

33. Langlois JA, Keyl PM, Guralnik JM et al. Characteristics of older pedestrians who have difficulty crossing the street. Am J Public Health 1997;87:393-397. 